(c) 2012 IEEE. Personal use of this material is permitted. Permission from IEEE must be obtained for all other uses, in any current or future media, including reprinting/republishing this material for advertising or promotional purposes, creating new collective works, for resale or redistribution to servers or lists, or reuse of any copyrighted component of this work in other works. 


\section{Characterizing mutual impedance of conical spiral antenna array for SKA radio telescope}

\author{
Shantanu K Padhi \\ CIRA/ICRAR, Curtin University \\ Perth, Australia \\ Email: shantanu.padhi@icrar.org
}

\author{
Hon Tat Hui \\ Department of Electrical and Computer Engineering, \\ National University of Singapore, Singapore \\ Email: elehht@nus.edu.sg
}

\begin{abstract}
This paper presents a detail analysis of conical spiral antenna on dispersive soil for SKA radio telescope application. An accurate formulation for calculation of mutual coupling effects in conical spiral antenna is introduced. The effects of mutual coupling are characterized in terms of design parameter. The mutual coupling formulation can be extended to include the effects in array configurations.
\end{abstract}

Keywords-conical spiral antenna; dispersive soil; radio astronomy; mutual coupling effect; mutual impedance.

\section{INTRODUCTION}

The Square Kilometre Array (SKA) is being developed as a radio telescope with unprecedented sensitivity, resolution and survey speed [1]. The SKA will have about two orders of magnitude improvement in sensitivity over existing facilities and many times improvement in the survey speed for better sky coverage. The primary objective of this instrument is to deliver few scientific key objectives such as to map the distribution of neutral hydrogen gas in the Universe, to study the origin of magnetic fields and to measure the dynamics of pulsars in the Galaxy. To realize these objectives, a very large collecting area is needed (more than one square kilometer) using new antenna technology and which can be ready for mass production with minimum cost. The SKA will be realized in two stages, Phase I $\left(\mathrm{SKA}_{1}\right)$ and Phase II $\left(\mathrm{SKA}_{2}\right)$. $\mathrm{SKA}_{1}$ will use sparse aperture arrays (AA-low) for the lowest band $(70-450 \mathrm{MHz})$ [2]. The antenna usually transforms the free-space energy to an RF signal, which is digitized and further processed using cross-correlation technique to reduce noise. The synthesized signal is used to reconstruct the spectral distribution of sky [1]. The AA-low will consist of several stations and each station will have broadband antenna elements. The size of station will also limit the field-of-view $(\mathrm{FoV})$ and the number of signals to be cross-correlated in signal processing algorithm. A total of 50 stations are specified for the SKA Phase I.

The conical spiral belongs to the frequency independent class of antennas. The frequency independent antennas, introduced by Rumsey [2], are those whose near-field and farfield characteristics are independent of frequency [2-3]. The conical log spiral antenna has desirable broadband features such as constant beam-width, slowly varying impedance, low cross polarization and low mutual coupling. They have been used in low frequency arrays such as Clark lake array [3]. The slowly varying impedance makes them easier to match to the LNA.

Broadband sparse arrays will have the lowest frequency affecting the antenna dimensions which in turn will dictate the minimum separation between individual antenna elements. At higher frequencies, this separation will amount to several wavelengths, which will reduce the filling factor. The physical conformability and relatively small linear dimensions of the spiral antenna help in reducing the minimum separation, reducing the filling factor deficiencies at higher frequencies. Other characteristics of the antenna are: constant beam patterns with frequency, high front-to-back (F/B) ratio, consistent impedance behaviour over the operating band, low ellipticity (enabling the polarization purity over the wide frequency band) [3] and low mutual coupling in an array configuration. The high gain of the antenna is also an important characteristic as higher gain implies fewer elements to reach a given array sensitivity.

In antenna array theory, mutual coupling between the antennas are highly undesired effect which drastically reduces

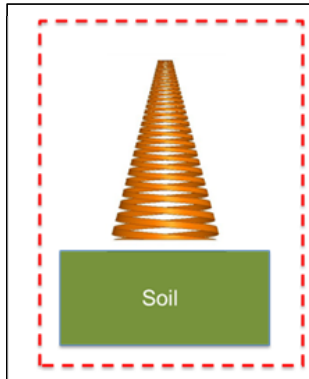

(a)

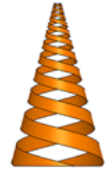

(b)
Fig. 1. (a) The geometry of conical spiral antenna, (b): the antennas in receiving mode with plane wave as source.

the performance or sensitivity of array (FoM). As discussed earlier [4-5], the mutual coupling for antenna array in transmitting mode is different than in receiving mode. For receiving array, the sensitivity relies heavily on accurate knowledge of terminal receive voltage and currents at each 
antenna feed point. All receiving array will have the mutual coupling effect which is already included in receive signals. Depending upon the frequency band relative to inter-element spacing, mutual coupling effects become critical in evaluating the performance of receive array. Hence, it is important to characterize and quantify the mutual coupling effects in receive array.

\section{RESULTS AND DISCUSSIONS}

This paper presents the design and development of circularly-polarized spiral antenna on dispersive soil (as shown in Fig. 1(a)) for SKA-low radio telescope application. For single-polarized antennas (either LHCP or RHCP), the wrap and cone angle are optimized to obtain smooth terminal characteristics and consistent radiation patterns over the frequency band in the $70-450 \mathrm{MHz}$ range, the $10 \mathrm{~dB}$ return loss bandwidth encompassing the whole range. The antenna shows a smooth impedance behavior over the frequency band with different moisture contents in soils (Fig. 2(a)). Calculated radiation patterns at $70 \mathrm{MHz}$ and $450 \mathrm{MHz}$ for both $\phi=0^{\circ}$ and $\phi=90^{\circ}$ are shown in Fig. 2(b-e). Back-lobes are prominent at lower frequencies only and cross-polar components are usually $20 \mathrm{~dB}$ or higher below the co-polar levels. The antenna exhibits moderate gain $\sim 6 \mathrm{~dB}$ over the operating frequency band.

\section{A. Mutual coupling effects;}

The geometry of antenna in receive mode configuration is shown in Fig 1(b), where a plane wave is used as source. Considering the plane wave type excitation, the induced voltages at both antenna terminals are calculated. The detail procedures for calculation of mutual impedance are outlined in [4-6]. The expression for mutual impedance is given in equation (1).

$$
Z^{12}=\frac{\left(S_{21 \_1}^{\prime}-S^{\prime \prime}{ }_{21 \_1}\right) Z_{l}}{S_{21 \_2}}
$$

The details of equation (1) are described in [5]. The coupling coefficients $(\mathrm{dB})$ of spiral antenna with respect to inter-element spacing as a parameter of wrap angle are shown in Fig. 2(f). As can be seen the coupling decreases as the spacing increases (increasing wrap angle). As can be seen in figure, for spacing $0.5 \lambda$ or less, the mutual coupling was very dominant. Mutual coupling will have significant effects in dense regime compared to sparse regime.

\section{CONCLUSIONS}

This paper presents a detail investigation of conical spiral antenna on dispersive soil for radio telescope application. The antenna exhibits linear impedance behavior over the frequency band. The minimum variations of terminal impedances with soil moisture contents are seen. The calculated radiation patterns are broadside back lobes are visible at lower frequency end only. The cross-polar components are fairly low and on average $20 \mathrm{~dB}$ below the co-polar levels. The mutual couplings effects are characterized with respect to design parameters of antenna. As the wrap angle increases the coupling decreases rapidly. (a)

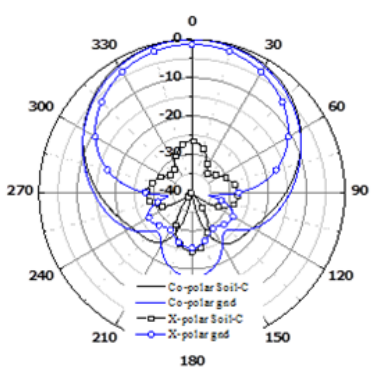

(c)

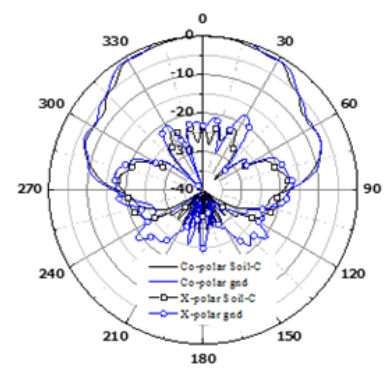

(e)

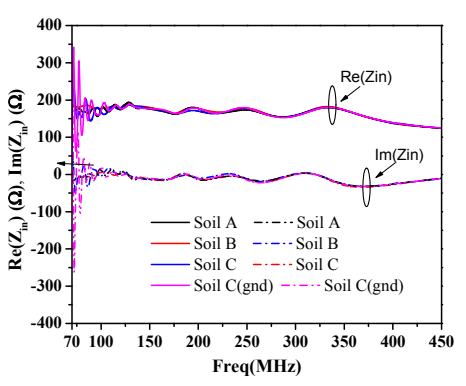

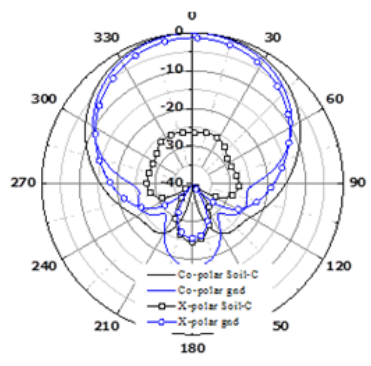

(b)

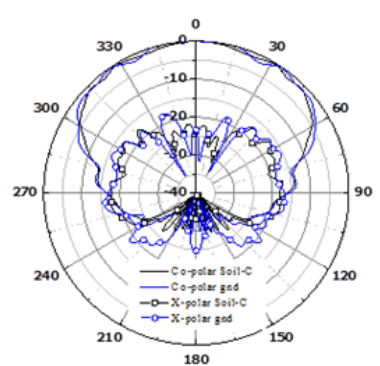

(d)

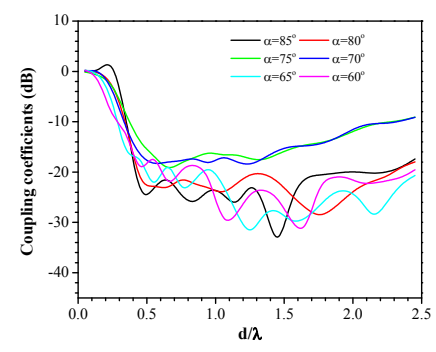

(f)
Fig 2. Calculated (a): terminal impedance of spiral antenna with dispersive soils; (b): radiation pattens at $70 \mathrm{MHz}$ in $\phi=0^{\circ}$ plane; (c): radiation pattens at $70 \mathrm{MHz}$ in $\phi=90^{\circ}$ plane; (d): radiation pattens at $450 \mathrm{MHz}$ in $\phi=0^{\circ}$ plane; (e): radiation pattens at $450 \mathrm{MHz}$ in $\phi=90^{\circ}$ plane; and (f): mutual coupling effects of spiral antenna.

\section{REFERENCES}

[1] P. Dewdney, P. Hall, R. T. Schillizzi, and T. J. L. W. Lazio, "The square kilometre array," Proceedings of IEEE, vol: 97, pp: 1482-1499, 2009.

[2] J. D. Dyson, “The Unidirectional Equiangular Spiral," IRE Transactions on Antennas and Propagation, vol:7, pp. 329-334, 1959.

[3] J. D. Dyson, "The characteristics and design of the conical log-spiral antenna," IRE Transactions on Antennas and Propagation, vol:13, pp. 488-499, July 1965.

[4] H. T. Hui, "A new definition of mutual impedance for application in dipole receiving antenna arrays" IEEE Transactions on Antennas and Propagation, vol:3, pp. 364-367, March 2004.

[5] H. T. Hui, K. Y. Chan and E. K. N. Yung, "Compensating for the mutual coupling effect in a normal-mode helical antenna array for adaptive nulling" IEEE Transactions on Vehicular Technol. vol:52, pp. 743-751, July 2003.

[6] C. A. Balanis, "Advanced engineering electromagnetics", New York, Wiley, 1989. 